\title{
Capacity and Error Performance of Reduced-Rank Transmitter Multiuser Preprocessing Based on Minimum Power Distortionless Response
}

\author{
Lie-Liang Yang, Senior Member, IEEE
}

\begin{abstract}
In this contribution we first derive a transmitter multiuser preprocessing (TMP) scheme for a general multiuser multiple-input-multiple-output (MIMO) system based on the minimum power distortionless response (MPDR) criterion, which minimizes the power in the context of a given downlink mobile terminal (MT) under the distortionless condition. This optimization problem results in a solution, which has the same form as the minimum mean-square error (MMSE) multiuser detection (MUD). Hence, we then extend the well-known rank-reduction techniques in MMSE-MUD to the MPDR-TMP in order to mitigate the possible implementation problems of the MPDRTMP. In our study three classes of rank-reduction algorithms are considered, which are derived, respectively, based on the eigen-analysis methods of principal components (PC) and crossspectral metrics (CSMs) as well as on the Taylor polynomial approximation (TPA) approach, which does not depend on the eigen-analysis. In this contribution both the capacity and error performance of a downlink space-division multiple-access (SDMA) system is investigated, when either the full-rank or reduced-rank MPDR-TMP is invoked. From our study and simulation results, it can be shown that the MPDR-TMP scheme is highly efficient for achieving the capacity and for suppressing the multiuser interference (MUI). The reduced-rank techniques can be employed by the MPDR-TMP, so as to reduce its implementation complexity while achieving the near full-rank performance of the full-rank MPDR-TMP.
\end{abstract}

Index Terms-Preprocessing, precoding, multiple-input multiple-output, space-division multiple-access, minimum power distortionless response, reduced-rank, subspace.

\section{INTRODUCTION}

$\mathbf{R}$ ECENTLY, transmitter multiuser preprocessing (TMP) or simply preprocessing, which suppresses multiuser interference by carrying out corresponding signal processing at the transmitter, has received wide attention [1-7]. One of the main advantages of employing the TMP techniques is that it is possible to implement low-complexity and high powerefficiency mobile terminals (MTs) for downlink transmissions in cellular systems using, typically, the time-division duplex (TDD) $[2,4]$. In the context of the preprocessing algorithms for TMP, it can be found from the literature that three typical optimization schemes have so far been invoked for deriving the preprocessing matrices, which include the matched-filtering

Manuscript received July 3, 2007; revised December 28, 2007; accepted March 18, 2008. The associate editor coordinating the review of this paper and approving it for publication was J. R. Luo.

The author is with the School of Electronics and Computer Science, University of Southampton, SO17 1BJ, UK, Tel: +44-23-8059 3364 (e-mail: 1ly@ecs.soton.ac.uk).

Digital Object Identifier 10.1109/T-WC.2008.070724
(MF) $[2,6,8,9]$, zero-forcing (ZF) or decorrelating [1, 3, 7, 10] as well as the minimum mean-square error (MMSE) [3-5, 11-13]. It can be shown that, when the MMSE-TMP does not exploit the knowledge about the noise power in the preprocessing, the MMSE-TMP scheme is then reduced to the ZF-TMP scheme $[3,13]$.

In this contribution another type of TMP scheme is proposed for preprocessing in general multiple-input-multipleoutput (MIMO) systems. The preprocessing algorithm is derived based on the minimum power distortionless response (MPDR) criterion, which minimizes the power in the context of a given downlink MT, while satisfying the distortionless condition. In principle, this optimization problem results in that the interference power imposed by the given MT on the other MTs is minimized [14]. From this point of view, the optimization does not seek to maximize the desired MT's performance, but tries to minimize the desired MT's effect on its colleague MTs. Hence, this type of optimization can be referred to as the altruistic (A)-optimization and hence the corresponding solution is A-optimum [14]. Although Aoptimization does not seek an optimum solution for the desired MT, however, as shown in [14] the overall solutions or the system is optimum, when all the invoked downlink MTs achieve their A-optimum solutions.

Specifically, in this contribution our study shows that the Aoptimization for TMP based on the MPDR criterion yields an A-optimum solution, which has the same form as the MMSE solution in MUD [15]. Hence, in a straightforward way, various classes of rank-reduction algorithms that are well-known in reduced-rank MMSE-based detections (see e.g., [16-24]) may be extended to the MPDR-TMP, in order to mitigate the possible problems in implementation of the MPDR-TMP, such as high-complexity and existence of ill-conditioned matrices that are not invertible. Note that, reduced-rank techniques have drawn wide attention for signal detection in various scenarios. However, the reduced-rank techniques have so far received little research in the context of transmitter preprocessing. Therefore, in this contribution three classes of rank-reduction algorithms are considered associated with the MPDR-TMP. These rank-reduction algorithms are derived, respectively, based on the eigen-analysis methods of principal components (PC) and cross-spectral metrics (CSM) $[16,18,19]$ as well as on the Taylor polynomial approximation (TPA) approach [15, 24], which does not depend on the eigen-analysis.

In this contribution both the capacity and error performance 
of the multiuser MIMO systems are investigated, when the proposed MPDR-TMP using either the full-rank or reducedrank preprocessing is employed. As an example, in our simulations the capacity and error performance of a downlink SDMA system is investigated. From our study and simulation results, it can be shown that the MPDR-TMP scheme is highly efficient for achieving the capacity of a MIMO system and also for suppressing the downlink MUI. The reduced-rank techniques can be efficiently employed by the MPDR-TMP, in order to reduce its implementation complexity while achieving the near full-rank performance.

The rest of the paper is organized as follows. Section II provides the preliminaries of a multiuser MIMO system, which assumes that the uplink and downlink channels are reciprocal. Section III derives the full-rank MPDR-TMP and its capacity expression. In Section IV three types of rankreduction techniques are introduced to the MPDR-TMP. In Section V simulation results in the context of both the capacity and bit error rate (BER) of the SDMA system are presented. Finally, in Section VI conclusions are summarized.

\section{Multiuser MiMO Models}

This contribution considers a general MIMO system having the downlink MIMO equation expressed as ${ }^{1}$

$$
\boldsymbol{y}_{D}=\boldsymbol{H}^{T} \boldsymbol{P} \boldsymbol{x}+\boldsymbol{n}=\boldsymbol{H}^{T} \sum_{k=1}^{K} \boldsymbol{p}_{k} x_{k}+\boldsymbol{n}
$$

where $\boldsymbol{y}_{D}$ is a $K$-length vector, $\boldsymbol{x}=\left[x_{1}, x_{2}, \ldots, x_{K}\right]^{T}$ contains the $K$ symbols conveyed by the downlink to one MT in point-to-point multiplexing scenario, or to upto $K$ MTs when a multiuser MIMO system, such as code-division multipleaccess (CDMA) or space-division multiple-access (SDMA), is considered. It is assumed that $E\left[\left|x_{k}\right|^{2}\right]=1$. In (1) $\boldsymbol{H}$ is the $(N \times K)$-dimensional channel transfer matrix given by

$$
\boldsymbol{H}=\left[\boldsymbol{h}_{1}, \boldsymbol{h}_{2}, \ldots, \boldsymbol{h}_{K}\right]
$$

where $\boldsymbol{h}_{k}$ is the channel impulse response (CIR) connecting the transmitter with the $k$ th $\mathrm{MT}$ and is hence referred to as the $k$ th user's signature. We assume that the entries $\left\{h_{i j}\right\}$ in $\boldsymbol{H}$ satisfy $E\left[\left|h_{i j}\right|^{2}\right]=1$. In (1) $\boldsymbol{P}$ is the $(N \times K)$ transmitter preprocessing matrix, which is denoted by

$$
\boldsymbol{P}=\left[\boldsymbol{p}_{1}, \boldsymbol{p}_{2}, \ldots, \boldsymbol{p}_{K}\right]
$$

where $\boldsymbol{p}_{k}$ is for preprocessing $x_{k}$. Furthermore, in (1) $\boldsymbol{n}$ is the $K$-length noise vector containing the noise samples observed at the $K$ MTs. We assume that each entry of $\boldsymbol{n}$ is a complex Gaussian random variable with zero mean and a variance of $\sigma^{2} / 2=1 / 2$ SNR per dimension, where SNR denotes the downlink signal-to-noise ratio (SNR) per MT.

Given the downlink MIMO equation of (1), it can be shown that the corresponding reciprocal uplink MIMO equation can be expressed as

$$
\boldsymbol{y}_{U}=\boldsymbol{H} \boldsymbol{x}+\boldsymbol{n}
$$

\footnotetext{
${ }^{1}$ Note that, more complicated MIMO equations can in general be simplified to the form of (1). For example, for the MIMO systems having processing at the MTs, the downlink MIMO equation can be expressed as $\boldsymbol{y}_{D}=\boldsymbol{W}_{D}^{H} \tilde{\boldsymbol{H}}^{T} \boldsymbol{P} \boldsymbol{x}+\boldsymbol{W}_{D}^{H} \tilde{\boldsymbol{n}}$, where $\boldsymbol{W}_{D}$ is the processing matrix at the MTs. Explicitly, this MIMO equation can be represented by (1) if we set $\boldsymbol{H}=\tilde{\boldsymbol{H}} \boldsymbol{W}_{D}^{*}$ and $\boldsymbol{n}=\boldsymbol{W}_{D}^{H} \tilde{\boldsymbol{n}}$.
}

where $\boldsymbol{y}_{U}$ is a $N$-length uplink observation vector, while $\boldsymbol{n}$ contains the noise observations of the uplink. Let assume that the symbols in $x$ are independent. Then, it can be shown that the auto-correlation matrix of $\boldsymbol{y}_{U}$ can be expressed as

$$
\begin{aligned}
\boldsymbol{R}_{U}=E\left[\boldsymbol{y}_{U} \boldsymbol{y}_{U}^{H}\right] & =\boldsymbol{H} \boldsymbol{H}^{H}+\sigma^{2} \boldsymbol{I}_{N} \\
& =\sum_{k=1}^{K} \boldsymbol{h}_{k} \boldsymbol{h}_{k}^{H}+\sigma^{2} \boldsymbol{I}_{N}
\end{aligned}
$$

when we assume that both the uplink and downlink have the same SNR value. It can be seen in our forthcoming discourse that $\boldsymbol{R}_{U}$ is useful for determining the subspace for the reducedrank TMP.

Note that, the general MIMO model considered in this section, as shown in (1) for downlink and (4) for uplink, may be used for representation of various wireless communications schemes. Specifically, the following three communications schemes have their uplink representation as (4) and their downlink representation as (1): (a) the point-to-point multiantenna MIMO system employing $(N \times K)$ antennas; (b) the SDMA system employing $N$ base-station (BS) antennas and supporting $K$ MTs each with one antenna; and (c) the CDMA system - where the BS employs one antenna and each MT also employs one antenna - using a spreading factor of $N$ and supporting $K$ users when communicating over flat fading channels. Let us first consider the full-rank MPDR-TMP in the next section.

\section{Full-Rank Transmitter Multiuser PREPROCESSING}

In this section we derive the preprocessing matrix $\boldsymbol{P}$ for the full-rank MPDR-TMP under the MPDR criterion, i.e., the full-rank MPDR-TMP. Specifically, the preprocessing vector $\boldsymbol{p}_{k}, k=1, \ldots, K$ is optimized, so that the $k$ th MT imposes the minimum interfering power on the other $(K-1)$ colleague MTs, while achieving its distortionless criterion. Again, we refer to this type of optimization, which minimizes the impact of the desired MT on the other MTs, as the A-optimization, and to the corresponding solution as A-optimum. Note that, conventionally, as shown, e.g., in [1-6], the optimization of preprocessing usually aims at deriving the preprocessing matrix $\boldsymbol{P}$ as seen in (3), instead of aiming at its component preprocessing vectors $\left\{\boldsymbol{p}_{k}\right\}$. The optimization concerning the component preprocessing vectors $\left\{\boldsymbol{p}_{k}\right\}$ has only been considered in [14] by the author of this contribution.

According to (1), it can be shown that the power related to $p_{k}$ plus the noise power in the context of the $k$ th MT can be expressed as

$$
\begin{gathered}
P_{\text {ower }}(k)=\operatorname{Tr}\left(\boldsymbol{p}_{k}^{H} \boldsymbol{H}^{*} \boldsymbol{H}^{T} \boldsymbol{p}_{k}\right)+\sigma^{2} \\
=\left|\boldsymbol{h}_{k}^{T} \boldsymbol{p}_{k}\right|^{2}+\sum_{l \neq k}^{K}\left|\boldsymbol{h}_{l}^{T} \boldsymbol{p}_{k}\right|^{2}+\sigma^{2}, \\
k=1,2, \ldots, K
\end{gathered}
$$

where $\operatorname{Tr}(\boldsymbol{A})$ denotes the trace of the square matrix $\boldsymbol{A}$. In the second equation of (6) the first term represents the power conveyed to the $k$ th (desired) MT, while the second term represents the interference imposed by the $k$ th MT on the 
other $(K-1)$ MTs, where the interference imposed by the $k$ th MT on the $l$ th MT is $\left|\boldsymbol{h}_{l}^{T} \boldsymbol{p}_{k}\right|^{2}$.

When the transmitter preprocessing is employed, the powerconstraint on the transmitted signals should be satisfied, which requires that the total transmission power after the preprocessing should not be increased. In this contribution we assume for simplicity that the power transmitted to each of the MTs maintains unchanged before and after the transmitter preprocessing. This implies that the preprocessing vector $\boldsymbol{p}_{k}$ in (1) should be chosen to satisfy

$$
\left\|\boldsymbol{p}_{k}\right\|^{2}=1, k=1,2, \ldots, K
$$

Consequently, upon invoking the power constraint, (6) can be modified to

$$
\begin{array}{r}
P_{\text {ower }}(k)=\operatorname{Tr}\left(\boldsymbol{p}_{k}^{H} \boldsymbol{H}^{*} \boldsymbol{H}^{T} \boldsymbol{p}_{k}\right)+\rho \sigma^{2}\left\|\boldsymbol{p}_{k}\right\|^{2} \\
=\operatorname{Tr}\left(\boldsymbol{p}_{k}^{H}\left[\boldsymbol{H}^{*} \boldsymbol{H}^{T}+\rho \sigma^{2} \boldsymbol{I}_{N}\right] \boldsymbol{p}_{k}\right), \\
k=1,2, \ldots, K
\end{array}
$$

where we deliberately applied a parameter $\rho \geq 0$ associated with the noise variance. We refer to $\rho$ as the noise-suppression factor, since its value is related to the noise suppression by the transmitter preprocessing. More details about the noisesuppression factor can be found in [14] and Section V, when showing the related simulation results.

Let define

$$
\boldsymbol{R}_{D}=\boldsymbol{H}^{*} \boldsymbol{H}^{T}+\rho \sigma^{2} \boldsymbol{I}_{N}=\sum_{k=1}^{K} \boldsymbol{h}_{k}^{*} \boldsymbol{h}_{k}^{T}+\rho \sigma^{2} \boldsymbol{I}_{N}
$$

Then, we have

$$
P_{\text {ower }}(k)=\operatorname{Tr}\left(\boldsymbol{p}_{k}^{H} \boldsymbol{R}_{D} \boldsymbol{p}_{k}\right), k=1,2, \ldots, K
$$

Notice that, when $\rho=1$, we have $\boldsymbol{R}_{D}=\boldsymbol{R}_{U}^{*}$. Hence, in practice when the uplink and downlink channels are reciprocal, the auto-correlation matrix $\boldsymbol{R}_{D}$ for downlink preprocessing can be estimated directly from the observations of the uplink channels using the well-known approaches, such as those in $[16,25]$. Specifically, let $\sigma_{U}^{2}$ be the noise variance of the uplink. Then, once the auto-correlation matrix $\boldsymbol{R}_{U}$ of the uplink has been estimated, the auto-correlation matrix $\boldsymbol{R}_{D}$ for downlink preprocessing can be formed as

$$
\boldsymbol{R}_{D}=\boldsymbol{R}_{U}^{*}-\left(\sigma_{U}^{2}-\rho \sigma^{2}\right) \boldsymbol{I}_{N}
$$

For transmitter preprocessing, the criterion of distortionless response can be expressed as

$$
\boldsymbol{h}_{k}^{T} \boldsymbol{p}_{k}=\beta_{k}
$$

where $\beta_{k}>0$ is for achieving the power constraint. Notice that, based on (6) (or (10)) and (11), given that the distortionless condition of (11) is satisfied, minimizing $P_{\text {ower }}(k)$ of (6) (or (10)) results in that the compound power of the interference imposed by the $k$ th MT on the other $(K-1)$ MTs and the background noise is minimized. Consequently, using the Lagrange multiplier, we can form the minimization problem for the MPDR-TMP as

$$
\begin{array}{r}
J=\boldsymbol{p}_{k}^{H} \boldsymbol{R}_{D} \boldsymbol{p}_{k}-\lambda^{*}\left(\boldsymbol{h}_{k}^{T} \boldsymbol{p}_{k}-\beta_{k}\right)-\left(\boldsymbol{p}_{k}^{H} \boldsymbol{h}_{k}^{*}-\beta_{k}\right) \lambda, \\
k=1,2, \ldots, K
\end{array}
$$

Upon taking the complex gradient of $J$ with respect to $\boldsymbol{p}_{k}^{*}$ and solving it, it gives

$$
\boldsymbol{p}_{k}=\boldsymbol{R}_{D}^{-1} \boldsymbol{h}_{k}^{*} \lambda
$$

where $\lambda$ can be obtained by substituting (13) into (11), yielding $\lambda=\beta_{k} /\left(\boldsymbol{h}_{k}^{T} \boldsymbol{R}_{D}^{-1} \boldsymbol{h}_{k}^{*}\right)$. Explicitly, the term $\boldsymbol{h}_{k}^{T} \boldsymbol{R}_{D}^{-1} \boldsymbol{h}_{k}^{*}$ can be absorbed into the power normalization factor. Consequently, the optimum preprocessing vector $\boldsymbol{p}_{k}$ in MPDR sense can be expressed as

$$
\boldsymbol{p}_{k}=\boldsymbol{R}_{D}^{-1} \boldsymbol{h}_{k}^{*} \bar{\beta}_{k}, k=1,2, \ldots, K
$$

where $\bar{\beta}_{k}=\beta_{k} /\left(\boldsymbol{h}_{k}^{T} \boldsymbol{R}_{D}^{-1} \boldsymbol{h}_{k}^{*}\right)$. It can be shown that (14) takes the same form as the MMSE solution in multiuser detection [15]. Upon applying (14) into (7), it can be shown that we have

$$
\bar{\beta}_{k}=\left(\boldsymbol{h}_{k}^{T} \boldsymbol{R}_{D}^{-2} \boldsymbol{h}_{k}^{*}\right)^{-1 / 2}
$$

Hence, the optimum preprocessing vectors in MPDR sense are finally given by

$$
\boldsymbol{p}_{k}=\frac{\boldsymbol{R}_{D}^{-1} \boldsymbol{h}_{k}^{*}}{\sqrt{\boldsymbol{h}_{k}^{T} \boldsymbol{R}_{D}^{-2} \boldsymbol{h}_{k}^{*}}}, k=1,2, \ldots, K
$$

Given the preprocessing vectors for the $K$ downlink MTs as shown in (16), the decision variable for the $k$ th MT can be expressed as

$$
y_{D}^{(k)}=\boldsymbol{h}_{k}^{T} \boldsymbol{p}_{k} x_{k}+\sum_{l \neq k}^{K} \boldsymbol{h}_{k}^{T} \boldsymbol{p}_{l} x_{l}+n_{k}, k=1,2, \ldots, K
$$

where $n_{k}$ is a Gaussian random variable with zero-mean and a variance of $\sigma^{2} / 2=1 / 2 \mathrm{SNR}$ per dimension. Based on (17), the signal-to-interference-plus-noise ratio (SINR) for detection of $x_{k}$ is given by

$$
\gamma_{k}=\frac{1}{2} \frac{E^{2}\left[y_{D}^{(k)}\right]}{\operatorname{Var}\left[y_{D}^{(k)}\right]}=\frac{1}{2}\left|\boldsymbol{h}_{k}^{T} \boldsymbol{p}_{k}\right|^{2}\left(\sum_{l \neq k}^{K}\left|\boldsymbol{h}_{k}^{T} \boldsymbol{p}_{l}\right|^{2}+\sigma^{2}\right)^{-1}
$$

When applying (16) into the above equation, it yields the SINR for the full-rank MPDR-TMP, which is given by

$$
\begin{gathered}
\gamma_{k}=\frac{1}{2} \bar{\beta}_{k}^{2}\left|\boldsymbol{h}_{k}^{T} \boldsymbol{R}_{D}^{-1} \boldsymbol{h}_{k}^{*}\right|^{2}\left(\sum_{l \neq k}^{K} \bar{\beta}_{l}^{2}\left|\boldsymbol{h}_{k}^{T} \boldsymbol{R}_{D}^{-1} \boldsymbol{h}_{l}^{*}\right|^{2}+\sigma^{2}\right)^{-1} \\
=\frac{\left|\boldsymbol{h}_{k}^{T} \boldsymbol{R}_{D}^{-1} \boldsymbol{h}_{k}^{*}\right|^{2}}{2 \boldsymbol{h}_{k}^{T} \boldsymbol{R}_{D}^{-2} \boldsymbol{h}_{k}^{*}}\left(\sum_{l \neq k}^{K} \frac{\left|\boldsymbol{h}_{k}^{T} \boldsymbol{R}_{D}^{-1} \boldsymbol{h}_{l}^{*}\right|^{2}}{\boldsymbol{h}_{l}^{T} \boldsymbol{R}_{D}^{-2} \boldsymbol{h}_{l}^{*}}+\frac{1}{\mathrm{SNR}}\right)^{-1} \\
k=1,2, \ldots, K
\end{gathered}
$$

Furthermore, according to (14), (16) and (17), it can be shown that the desired power conveyed to the $k$ th MT can be expressed as

$$
\begin{aligned}
P_{\text {ower }}^{(D)}(k)=\left|\boldsymbol{h}_{k}^{T} \boldsymbol{p}_{k}\right|^{2} & =\bar{\beta}_{k}^{2}\left|\boldsymbol{h}_{k}^{T} \boldsymbol{R}_{D}^{-1} \boldsymbol{h}_{k}^{*}\right|^{2} \\
& =\frac{\left|\boldsymbol{h}_{k}^{T} \boldsymbol{R}_{D}^{-1} \boldsymbol{h}_{k}^{*}\right|^{2}}{\boldsymbol{h}_{k}^{T} \boldsymbol{R}_{D}^{-2} \boldsymbol{h}_{k}^{*}} .
\end{aligned}
$$

Straightforwardly, when given the transmission power in the context of $\boldsymbol{p}_{k}$, it is desirable that the desired power received 
by the $k$ th MT, i.e., $P_{o w e r}^{(D)}(k)$, is as high as possible, in order to minimize the interference of the $k$ th MT on the other MTs.

When assuming that $x_{k}, k=1, \ldots, K$ is taken from a zeromean Gaussian process with unity variance and that, after the MPDR-assisted optimum preprocessing, the decision variable $y_{D}^{(k)}$ in (17) can be approximated as a Gaussian variable with its corresponding SINR given by (19), the mutual information between $x_{k}$ and $y_{D}^{(k)}$ or the channel capacity corresponding to the $k$ th MT can be expressed as [26]

$$
\begin{array}{r}
\mathcal{C}_{k}=\frac{1}{2} E_{\gamma_{k}}\left[\log _{2}\left(1+2 \gamma_{k}\right)\right] \text { bits } / \mathrm{s}(\text { ample }) / \mathrm{Hz}, \\
k=1,2, \ldots, K
\end{array}
$$

In our forthcoming discourse we refer to (21) as the fullrank channel capacity per MT. According to (21), intuitively, an efficient reduced-rank MPDR-TMP scheme should be able to reach the full-rank channel capacity with a rank as low as possible. Let us now turn to consider the reduced-rank MPDRTMP in the next section.

\section{Reduced-Rank Transmitter Multiuser PREPROCESSING}

As shown in (16), in order to derive the preprocessing vector $\boldsymbol{p}_{k}$ for $k=1, \ldots, K$, the following main computations are required: (a) inverse the $(N \times N)$ matrix $\boldsymbol{R}_{D}$ once $^{2}$, which has a complexity on the order of $O\left(N^{3}\right)$; (b) $K\left(N^{2}+N\right)$ complex multiplications, where $N^{2}$ is from computing $\boldsymbol{R}_{D}^{-1} \boldsymbol{h}_{k}^{*}, N$ is from computing $\boldsymbol{h}_{k}^{T} \boldsymbol{R}_{D}^{-2} \boldsymbol{h}_{k}^{*}$. Hence, we can be implied that, when $N$ is large, the complexity for deriving the preprocessing vectors might be extreme. Additionally, in wireless communications especially in SDMA systems where user signatures are formed by CIRs, the covariance matrix $\boldsymbol{R}_{D}$ might be illconditioned and is not invertible. Although the probability is low, this may happen when two or more user signatures are highly correlated, or when one or several user signatures or their linear combination lies in the noise subspace. Therefore, in this section we investigate the transmitter preprocessing in reduced-rank subspaces, in order to reduce the complexity for computing the preprocessing vectors $\left\{\boldsymbol{p}_{k}\right\}$, and/or to circumvent the problem of ill-conditioned covariance matrix.

\section{A. General Theory}

The reduced-rank TMPs start with expressing the preprocessing vectors $\boldsymbol{p}_{k}$ as

$$
\boldsymbol{p}_{k}=\boldsymbol{P}_{k}^{*} \overline{\boldsymbol{p}}_{k}, k=1,2, \ldots, K
$$

where $\boldsymbol{P}_{k}$ is a $(N \times L)$ matrix, which is referred to as the processing matrix (preprocessing subspace) for convenience, while $\bar{p}_{k}$ is a $L$-length vector to be determined based on the MPDR optimization. It can be shown that, by substituting (22) into (12) and following the similar approaches for deriving $\boldsymbol{p}_{k}$,

\footnotetext{
${ }^{2}$ Note that, since in the proposed MPDR-TMP $\boldsymbol{R}_{D}$ is only required to be inverted once for all the MTs even in the full-rank scenario, the complexity of the proposed MPDR-TMP in full-rank case may still be substantially lower than that of the TMP based on the singular value decomposition (SVD) principles [10], where the SVD having a complexity of $O\left((N-1)^{3}\right)$ must be operated in the context of each of the MTs.
}

we can find the solution to $\overline{\boldsymbol{p}}_{k}$ in the $L$-rank subspace, which can be expressed as

$$
\overline{\boldsymbol{p}}_{k}=\left(\boldsymbol{P}_{k}^{T} \boldsymbol{R}_{D} \boldsymbol{P}_{k}^{*}\right)^{-1} \boldsymbol{P}_{k}^{T} \boldsymbol{h}_{k}^{*} \bar{\beta}_{k}, k=1,2, \ldots, K
$$

where $\bar{\beta}_{k}$ is for achieving the constraint on the transmission power. According to (7), $\bar{\beta}_{k}$ is given by

$$
\bar{\beta}_{k}=1 / \sqrt{\left\|\boldsymbol{P}_{k}^{*}\left(\boldsymbol{P}_{k}^{T} \boldsymbol{R}_{D} \boldsymbol{P}_{k}^{*}\right)^{-1} \boldsymbol{P}_{k}^{T} \boldsymbol{h}_{k}^{*}\right\|^{2}}
$$

With the aid of (22), (23) and (24), the SINR observed at MT $k$ for the reduced-rank TMP can be computed by (18). Furthermore, the mutual information between $x_{k}$ and $y_{D}^{(k)}$ for the reduced-rank TMP can be obtained from (21).

As shown in (23), instead of inverting a $(N \times N)$ matrix in (14) for the full-rank TMP, the reduced-rank TMP of (23) inverts a $(L \times L)$ matrix. Therfore, for design of reduced-rank MPDR-TMP, one of the main tasks is to derive a $L<N$ dimensional preprocessing subspace, so that it can approximate the $\mathrm{N}$-dimensional original space as closely as possible or so that the error performance achieved by the reducedrank MPDR-TMP can be similar as that achieved by the fullrank TMP. Below three types of rank-reduction algorithms are derived and investigated. These reduced-rank MPDR-TMP algorithms constitute the counterparts of the reduced-rank detection algorithms that have been widely studied in array processing and MUD, as seen, e.g., in [16-24] as well as the references in them. However, we note again that the reducedrank TMP has so far received relatively little attention in literature.

\section{B. Eigenspace: Principal Components}

The principal components (PCs) based reduced-rank signal processing has received wide research and application in array processing and signal detection $[16,17]$. With the PC-based approach, eigen-decomposition on the auto-correlation matrix is carried out and a number of principal eigenvectors are chosen to form a subspace, which is used to approximate the original space.

In the context of the PC-based reduced-rank MPDR-TMP, the Hermitian auto-correlation matrix $\boldsymbol{R}_{D}$ in (9) can be expressed with the aid of its eigen-decomposition as $^{3}$

$$
\boldsymbol{R}_{D}=\boldsymbol{R}_{U}^{*}=\boldsymbol{\Phi}^{*} \boldsymbol{\Lambda} \boldsymbol{\Phi}^{T}=\sum_{n=1}^{N} \lambda_{n} \boldsymbol{\phi}_{n}^{*} \boldsymbol{\phi}_{n}^{T}
$$

where $\Phi^{*}=\left[\phi_{1}^{*}, \boldsymbol{\phi}_{2}^{*}, \cdots, \boldsymbol{\phi}_{N}^{*}\right]$ is an orthonormal matrix whose columns consist of the eigenvectors of $\boldsymbol{R}_{D}$, where $\boldsymbol{\phi}_{n}^{*}$ is the eigenvector corresponding to the eigenvalue $\lambda_{n}, \boldsymbol{\Lambda}$ is a diagonal matrix containing the eigenvalues, ie.

$$
\boldsymbol{\Lambda}=\operatorname{diag}\left\{\lambda_{1}, \lambda_{2}, \ldots, \lambda_{N}\right\}
$$

Let us assume that the eigenvalues are ordered as $\lambda_{1} \geq$ $\lambda_{2} \geq \ldots \geq \lambda_{N}$. Then, for a given rank $L$ of the preprocessing subspace, the processing matrix $\boldsymbol{P}_{k}$ in (22) in the context of

\footnotetext{
${ }^{3}$ Below we deliberately invoke the MUD-related auto-correlation matrix $\boldsymbol{R}_{U}$ in our analysis, in order to emphasize the fact that the reduced-rank techniques can be directly based on $\boldsymbol{R}_{U}$, when the uplink and downlink channels are reciprocal.
} 
the PC-based reduced-rank MPDR-TMP is constituted by the first $L$ columns of $\Phi$, which can be expressed as

$$
\boldsymbol{P}_{k}=\boldsymbol{P}_{L}=\left[\boldsymbol{\phi}_{1}, \boldsymbol{\phi}_{2}, \ldots, \boldsymbol{\phi}_{L}\right], k=1,2, \ldots, K
$$

Explicitly, $\boldsymbol{P}_{k}$ is independent of the MT index of $k$ and $\boldsymbol{P}_{k}=$ $P_{L}$ is hence the same for all the MTs. Consequently, when the PC-based reduced-rank MPDR-TMP is considered, we only need to compute the preprocessing subspace $\boldsymbol{P}_{k}=\boldsymbol{P}_{L}$ once as well as the inverse operation $\left(\boldsymbol{P}_{k}^{T} \boldsymbol{R}_{D} \boldsymbol{P}_{k}^{*}\right)^{-1}$ in (23) once, in order to derive the $K$ preprocessing vectors for the $K$ MTs. Therefore, it can be implied that the PC-based reduced-rank MPDR-TMP has a low implementation complexity. However, as our simulation results in Fig. 7 of Section V shown, unless the preprocessing subspace has the same rank as the signal subspace, ie., unless $L=K$, the downlink SDMA otherwise conflicts severe MUI and the error performance of the PCbased reduced-rank MPDR-TMP is much worse than that of the full-rank MPDR-TMP.

Upon applying (27) into (23) and (22), we can find that the preprocessing vectors for the PC-based reduced-rank MPDRTMP can be expressed as

$$
\begin{aligned}
\boldsymbol{p}_{k} & =\boldsymbol{P}_{L}^{*} \overline{\boldsymbol{p}}_{k}=\boldsymbol{P}_{L}^{*}\left(\sum_{n=1}^{N} \lambda_{n} \boldsymbol{P}_{L}^{T} \boldsymbol{\phi}_{n}^{*} \boldsymbol{\phi}_{n}^{T} \boldsymbol{P}_{L}^{*}\right)^{-1} \boldsymbol{P}_{L}^{T} \boldsymbol{h}_{k}^{*} \bar{\beta}_{k} \\
& =\boldsymbol{P}_{L}^{*} \boldsymbol{\Lambda}_{L}^{-1} \boldsymbol{P}_{L}^{T} \boldsymbol{h}_{k}^{*} \bar{\beta}_{k}, k=1,2, \ldots, K
\end{aligned}
$$

where $\Lambda_{L}^{-1}=\operatorname{diag}\left\{\lambda_{1}^{-1}, \lambda_{2}^{-1}, \ldots, \lambda_{L}^{-1}\right\}$, and $\bar{\beta}_{k}=$ $1 / \sqrt{\left|\boldsymbol{h}_{k}^{T} \boldsymbol{P}_{L}^{*} \boldsymbol{\Lambda}_{L}^{-2} \boldsymbol{P}_{L}^{T} \boldsymbol{h}_{k}^{*}\right|}$.

\section{Eigenspace: Cross-Spectral Metric}

The cross-spectral metric (CSM) based technique for reduced-rank signal detection has been investigated, e.g., in [16-18,20-23]. In the context of the CSM-based reducedrank MPDR-TMP, the processing matrix $\boldsymbol{P}_{k}, k=1, \ldots, K$ is formed as follows.

For the full-rank MPDR-TMP, the desired power transmitted to the $k$ th MT is given by (20). When applying (25) of the eigen-representation of the auto-correlation matrix $\boldsymbol{R}_{D}$ into (20), the desired power conveyed to the $k$ th MT can be expressed as

$$
P_{\text {ower }}^{(D)}(k)=\bar{\beta}_{k}^{2} \times\left|\sum_{n=1}^{N} \frac{\left|\boldsymbol{h}_{k}^{T} \boldsymbol{\phi}_{n}^{*}\right|^{2}}{\lambda_{n}}\right|^{2}
$$

In principle, given the constraint on the transmission power and the MPDR criterion, the preprocessing vector, say $\bar{p}_{k}$, in the preprocessing subspace should be designed so that the power conveyed to the $k$ th (desired) MT is as high as possible. This in turn implies that those $\phi_{n}$ 's that are most similar to the signature $h_{k}$ of the $k$ th MT should be chosen to form the processing matrix $\boldsymbol{P}_{k}$. Hence, given the dimension $L$ of the preprocessing subspace, the processing matrix $\boldsymbol{P}_{k}$ for the CSM-based reduced-rank MPDR-TMP is constituted by the $L$ eigenvectors of $\boldsymbol{R}_{D}^{*}$, which correspond with the largest $L$ values of the CSMs defined as

$$
\left|\boldsymbol{h}_{k}^{T} \boldsymbol{\phi}_{n}^{*}\right|^{2}, i=1, \ldots, N
$$

Note that, the difference between the CSM in TMP and that in MUD, see, e.g. $[18,20-23,27]$, is that the CSM in MUD takes into account the power in the direction of an eigenvector and is defined as $\left|\boldsymbol{h}_{k}^{T} \boldsymbol{\phi}_{n}^{*}\right|^{2} / \lambda_{n}$. The CSM defined in this way is capable of resulting in a minimum of the mean-square error (MSE) for a given rank $L$ of the subspace. By contrast, for our MPDR-TMP, given the constraint on the transmission power in terms of $\boldsymbol{p}_{k}$, i.e. $P_{\text {ower }}(k)$, it is straightforward that the better performance may be achieved, when more transmission power of $P_{\text {ower }}^{(D)}(k)$ is conveyed to the $k$ th (desired) MT. Hence, the definition of (30) is appropriate.

Finally, it can be shown that the $k$ th preprocessing vector $\boldsymbol{p}_{k}$ in the CSM-based reduced-rank MPDR-TMP has a similar form as (28), which can be expressed as

$$
\boldsymbol{p}_{k}=\boldsymbol{P}_{k}^{*} \boldsymbol{\Lambda}_{k}^{-1} \boldsymbol{P}_{k}^{T} \boldsymbol{h}_{k}^{*} \bar{\beta}_{k}, k=1,2, \ldots, K
$$

where $\boldsymbol{\Lambda}_{k}^{-1}=\operatorname{diag}\left\{\lambda_{k 1}^{-1}, \lambda_{k 2}^{-1}, \ldots, \lambda_{k L}^{-1}\right\}$ and its $L$ eigenvalues are in correspondence with the $L$ eigenvectors in $\boldsymbol{P}_{k}$.

When compare the CSM-based reduced-rank MPDR-TMP with the PC-based reduced-rank MPDR-TMP, it can be seen that in the CSM-based reduced-rank MPDR-TMP the processing matrix $\boldsymbol{P}_{k}$ is formed with considering the power conveyed to the desired MT. By contrast, the PC-based reduced-rank MPDR-TMP tries to approximate the auto-correlation matrix $\boldsymbol{R}_{D}$ regardless of the power conveyed to each of the remote MTs. From this point of view and also as shown by our simulation results in Figs. 7 and 8 of Section V, the CSM-based reduced-rank MPDR-TMP outperforms the PC-based reduced-rank MPDR-TMP in terms of their BER performance. However, in the PC-based reduced-rank MPDRTMP the preprocessing subspace, ie. $\boldsymbol{P}_{k}$, is the same for all the MTs, but in the CSM-based reduced-rank MPDR-TMP every MT corresponds to a specific $\boldsymbol{P}_{k}$. Hence, the implementation complexity of the PC-based reduced-rank MPDR-TMP can be lower than that of the CSM-based reduced-rank MPDR-TMP.

\section{Taylor Polynomial Approximation}

The processing matrix $\boldsymbol{P}_{k}(k=1, \ldots, K)$ in the Taylor polynomial approximation (TPA) assisted reduced-rank TMP is derived by first approximating the term of $\boldsymbol{R}_{D}^{*-1} \boldsymbol{h}_{k}$ in (14) with the aid of the Taylor expansion of the auto-correlation matrix $R_{D}$. The TPA technique has been originally applied for deriving the reduced-rank linear detectors for CDMA systems $[15,24]$. Specifically, let $\lambda_{\max }$ be the maximum eigenvalue of $\boldsymbol{R}_{D}^{*}=\boldsymbol{R}_{U}$. Let $\mu$ be a constant satisfying $0<\mu<1 / \lambda_{\max }$. Then, the matrix $\boldsymbol{R}_{D}^{*-1}$ can be Taylor expanded as $[15,24]$

$$
\begin{aligned}
\boldsymbol{R}_{U}^{-1}=\boldsymbol{R}_{D}^{*-1}=\mu\left(\mu \boldsymbol{R}_{D}^{*}\right)^{-1} & =\mu\left[\boldsymbol{I}-\left(\boldsymbol{I}-\mu \boldsymbol{R}_{D}^{*}\right)\right]^{-1} \\
& =\mu \sum_{n=0}^{\infty}\left(\boldsymbol{I}-\mu \boldsymbol{R}_{D}^{*}\right)^{n}
\end{aligned}
$$

Using the first $L$ terms in (32) to approximate $R_{D}^{*-1}$, we obtain

$$
\begin{aligned}
\boldsymbol{R}_{D}^{*-1} & \approx \mu \sum_{n=0}^{L-1}\left(\boldsymbol{I}-\mu \boldsymbol{R}_{D}^{*}\right)^{n} \\
& =a_{0} \boldsymbol{I}+a_{1} \boldsymbol{R}_{D}^{*}+\cdots+a_{L-1} \boldsymbol{R}_{D}^{* L-1}
\end{aligned}
$$

where the coefficients $\left\{a_{n}\right\}$ are determined by $\mu$ associated with the expansion of (33). Upon substituting (34) into (14), 
the $k$ th preprocessing vector can be approximately expressed as

$$
\begin{array}{r}
\boldsymbol{p}_{k} \approx\left(a_{0} \boldsymbol{h}_{k}+a_{1} \boldsymbol{R}_{D}^{*} \boldsymbol{h}_{k}+\ldots+a_{L-1} \boldsymbol{R}_{D}^{* L-1} \boldsymbol{h}_{k}\right)^{*} \bar{\beta}_{k}, \\
k=1,2, \ldots, K
\end{array}
$$

As shown in (35) there are $L$ number of coefficients that must be determined, which are depended on the expansion of (33) as well as the constant $\mu$ selected. Furthermore, the choosing of the constant $\mu$ depends on the eigen-decomposition of $\boldsymbol{R}_{D}^{*}$. Hence, determining the coefficients $\left\{a_{n}\right\}$ in (35) may result in a high complexity. Additionally, as noted in [28], the finite order approximations that result from tail-cutting of infinite order approximations generally do not lead to the best fit among all approximations of the same order. Hence, instead of using the approximation as in (35), we form a $(N \times L)$ processing matrix as

$$
\begin{aligned}
\boldsymbol{P}_{k} & =\left[\boldsymbol{h}_{k}, \boldsymbol{R}_{D}^{*} \boldsymbol{h}_{k}, \cdots, \boldsymbol{R}_{D}^{* L-1} \boldsymbol{h}_{k}\right] \\
& =\left[\boldsymbol{I}_{N}, \boldsymbol{R}_{D}^{*}, \cdots, \boldsymbol{R}_{D}^{* L-1}\right]\left(\boldsymbol{I}_{L} \otimes \boldsymbol{h}_{k}\right) \\
& =\boldsymbol{\mathcal { R }}_{L}\left(\boldsymbol{I}_{L} \otimes \boldsymbol{h}_{k}\right), \quad k=1,2, \ldots, K
\end{aligned}
$$

where $\boldsymbol{\mathcal { R }}_{L}=\left[\boldsymbol{I}_{N}, \boldsymbol{R}_{D}^{*}, \cdots, \boldsymbol{R}_{D}^{* L-1}\right]$ and $\otimes$ represents the Kronecker product [16] operation. With the processing matrix $\boldsymbol{P}_{k}$, then, the preprocessing vectors $\boldsymbol{p}_{k}$ for $k=1,2, \ldots, K$ can be computed according to (22) and (23).

In [29] a reduced-complexity precoding scheme has been investigated in the context of the frequency-selective MIMO channels, where a preprocessing subspace having a similar structure as (36) is applied. In [29] the preprocessing subspace has been derived from the extension of the multistage Wiener filter [21], which is a complicated mathematical framework for deriving the subspace of (36). Note that, the study in $[22,30]$ has shown that the TPA-based reduced-rank MMSE detector is equivalent to the reduced-rank MMSE detector based on the multistage Wiener filter [21].

It can be noticed from (36) that deriving $\boldsymbol{P}_{k}$ does not depend on the eigen-analysis. Furthermore, (36) shows that $\boldsymbol{R}_{L}$ is the same for all the MTs. Hence, although $\boldsymbol{P}_{k}$ needs to be computed with respect to each of the MTs, the transmitter however only requires to compute $\mathcal{R}_{L}$ once. Therfore, the TPAbased reduced-rank MPDR-TMP may even demand lower implementation complexity than the CSM-based reducedrank MPDR-TMP. Furthermore, since the Taylor polynomial expansion employs the property that the resulted sequence converges exponentially to the true value, as the simulation results in Figs. 9 and 10 shown, the TPA-based reduced-rank MPDR-TMP is capable of achieving the near full-rank error performance with a preprocessing subspace having a rank that may be significantly lower than the rank of the corresponding signal subspace.

\section{Performance Results}

In this section we provide a range of results in order to show the performance and characteristics of the MIMO systems using full- or reduced-rank MPDR-TMPs. Specifically, the capacity and BER performance of the SDMA downlink systems are considered. When the capacity is considered, we assume that the transmitted data symbol $x_{k}(k=1,2, \ldots, K)$

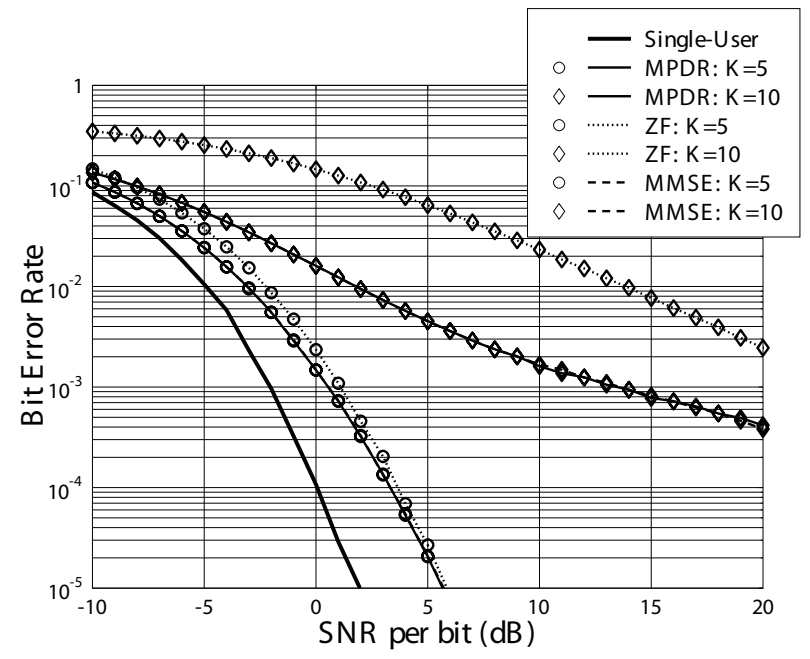

Fig. 1. Comparison of BER versus SNR per bit performance of the SDMA systems using MPDR, zero-forcing (ZF) and minimum mean-square error (MMSE) assisted transmitter preprocessing schemes, when communicating over Rayleigh fading channels. In the simulations a noise-suppression factor of $\rho=1$ was assumed.

obeys the Gaussian distribution. It is also assumed that the total transmission power maintains constant regardless of the number of MTs supported, in order to illustrate the effect of the number of MTs (or the number of receive antennas) on the achievable capacity. By contrast, when the BER performance is considered, we assume binary phase-shift keying (BPSK) baseband modulation, where $x_{k}(k=1,2, \ldots, K)$ takes a value of +1 or -1 with equal probability. Furthermore, when considering the BER performance, we assume that the transmission energy per bit is constant. Hence, when the SDMA supports more MTs, higher total transmission power is required. Additionally, in our simulations we assume that the channel connecting any of the transmit antennas with any of the MTs experiences independent flat Rayleigh fading.

In Fig. 1 we compare the BER versus SNR per bit performance of the SDMA systems, when the proposed MPDRTMP, zero-forcing TMP (ZF-TMP) [3] or the minimum meansquare error TMP (MMSE-TMP) [4,5] is employed. In our simulations the SDMA system employed $N=10$ BS transmitter antennas and the noise-suppression factor seen in (8) was set to $\rho=1$. From the results of Fig. 1 we can observe that the MPDR-TMP is capable of efficiently suppressing the downlink MUI; it is capable of achieving the same BER performance as the MMSE-TMP, and both the MPDR-TMP and MMSE-TMP outperform the ZF-TMP, especially when the number of MTs supported is high. Note that, as in MUD, it is not hard to prove that the MPDR-TMP and MMSE-TMP are equivalent in term of the achievable error rate performance, although they are derived based on different optimization principles. From the results of Fig. 1 we can observe that, there is a trade-off between the achievable BER and the number of MTs supported for all the three TMP schemes. The BER performance becomes worse when the number of MTs supported increases. The reason for this observation is that the diversity order decreases, when the number of MTs supported increases, as the BS transmitter has to invest a corresponding degrees-of-freedom for MUI suppression. 
Full-rank MPDR-TMP: $\mathrm{N}=10, \rho=1$, total Tx power=constant

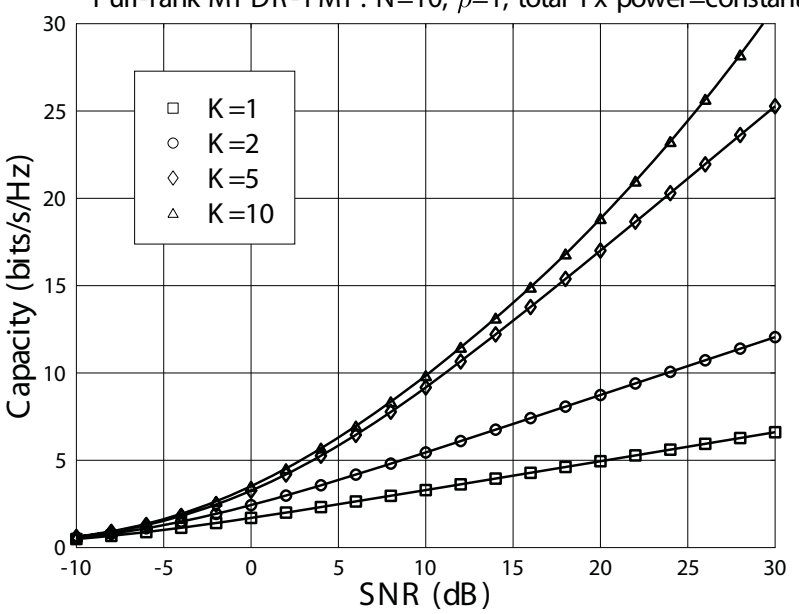

Fig. 2. Full-rank: Capacity versus SNR performance of the MPDRTMP assisted SDMA using $N=10$ BS transmit antennas and the noisesuppression factor of $\rho=1$, when communicating over Rayleigh fading channels and assuming that the total transmission power is constant regardless of the number of MTs supported.

Full-rank MPDR-TMP: $\mathrm{N}=20, \rho=1$, total Tx power=constant

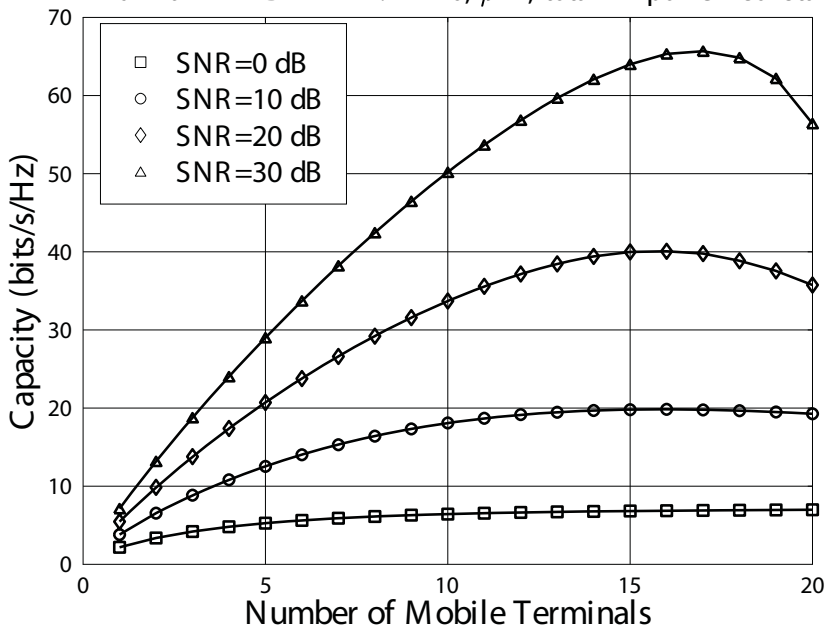

Fig. 3. Full-rank: Capacity versus number of MTs $(K)$ performance of the MPDR-TMP assisted SDMA using $N=20 \mathrm{BS}$ transmit antennas and the noise-suppression factor of $\rho=1$, when communicating over Rayleigh fading channels and assuming that the total transmission power is constant regardless of the number of MTs supported.

Fig. 2 shows the capacity of the SDMA system using $N=10 \mathrm{BS}$ transmit antennas and supporting $K=1,2,5,10$ MTs, when the full-rank MPDR-TMP is employed. Note again that, in our simulations for capacity, we assumed that the total transmission power and hence the total SNR did not scale with the number of MTs supported. In other words, a given SNR value seen in Fig. 2 retains constant no matter how many MTs are supported. The results of Fig. 2 show that the capacity increases, when the total transmission power or the total SNR increases. For the four $K$ values considered in Fig. 2, the capacity is higher, when the $K$ value is higher, ie., when the number of MTs supported is higher. However, this is not always correct, as shown in Fig. 3

In Fig. 3 the capacity of the SDMA system using $N=20$ $\mathrm{BS}$ transmit antennas is illustrated against the number of MTs, when the SNR is $0 \mathrm{~dB}, 10 \mathrm{~dB}, 20 \mathrm{~dB}$ or $30 \mathrm{~dB}$. From the results
Full-rank MPDR-TMP: $\mathrm{N}=20, \mathrm{SNR}=30 \mathrm{~dB}$, total $\mathrm{T} x$ power=constant

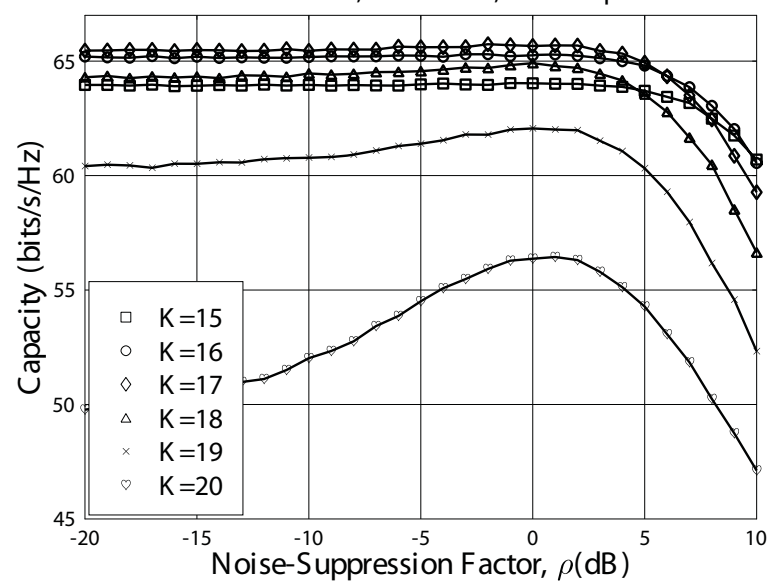

Fig. 4. Full-rank: Capacity versus noise-suppression factor $(\rho)$ performance of the MPDR-TMP assisted SDMA using $N=20$ BS transmit antennas, when communicating over Rayleigh fading channels and assuming that the total transmission power is constant regardless of the number of MTs supported.

of Fig. 3 we can have the following observations. Firstly, for a given SNR value, the capacity scales near-linearly with the number of MTs, when the number of MTs is relatively low. This observation is explicit, when the SNR value is high, such as when $\mathrm{SNR}=20 \mathrm{~dB}$ or $30 \mathrm{~dB}$. Secondly, for a given total SNR especially when this SNR value is relatively high, there exists an optimum number of MTs, which results in the highest capacity of the SDMA system. As seen in Fig. 3, after the optimum point, the capacity of the SDMA system decreases when the number of MTs supported further increases. Furthermore, as shown in Fig. 3, when the SNR value is higher, the linear increasing range of capacity with $K$ is also bigger. The reason for the SDMA system using MPDR-TMP to have the above-observed characteristics can be explained as follows. Given the total transmission power or a total SNR, the SDMA system's capacity increases with the number of MTs or receive antennas [31]. However, when the SDMA using MPDR-TMP supports more MTs, the power must be distributed to more MTs, resulting in that the SNR per MT decreases. In this contribution we assumed that the power was evenly distributed to the MTs, which is not optimum according to the information theory [26,31]. Additionally, when there are more MTs, each MT conflicts higher MUI, which results in that the SNR per MT further decreases. Therefore, the SDMA system's capacity decreases due to the decrease of the SNR per MT and to the non-ideal power assignment. Consequently, the above-mentioned positive and negative effects on the capacity result in that the SDMA system using MPDR-TMP has the capacity curves as shown in Fig. 3.

Fig.4 illustrates the effect of the noise-suppression factor $\rho$ on the capacity of the SDMA using full-rank MPDR-TMP, when the BS employs $N=20$ transmit antennas and the total SNR is $30 \mathrm{~dB}$. From the results of Fig.4 we can observe that the optimum value of the noise-suppression factor is around $\rho=1(0 \mathrm{~dB})$. Therefore, in our following simulations, we mainly used $\rho=1$. Additionally, it can be seen from Fig.4 that the capacity of the SDMA system is not highly 


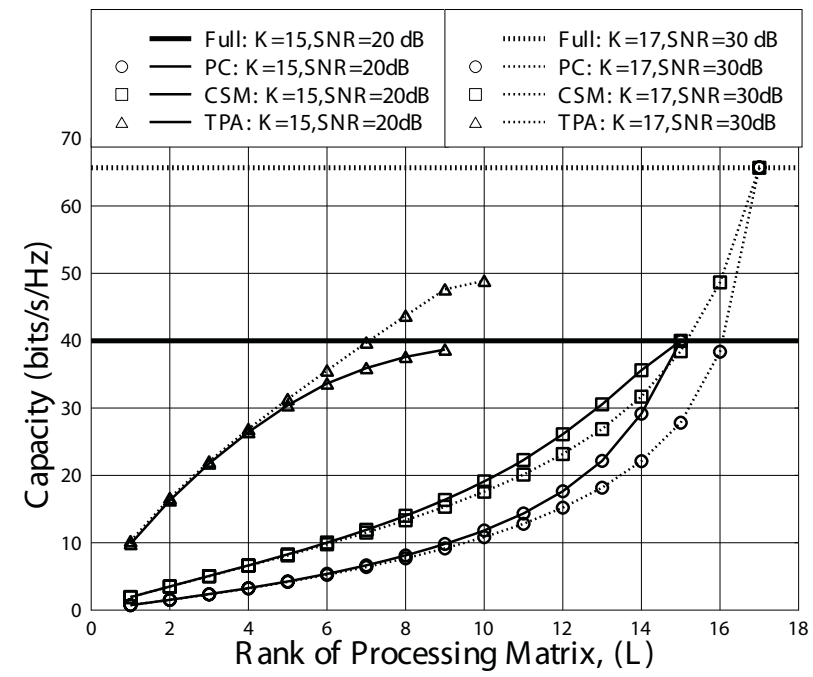

Fig. 5. Reduced-rank: Capacity versus rank $L$ performance of the MPDR-TMP assisted SDMA using various rank reduction schemes, when communicating over Rayleigh fading channels and assuming that the total transmission power is constant regardless of the number of MTs supported.

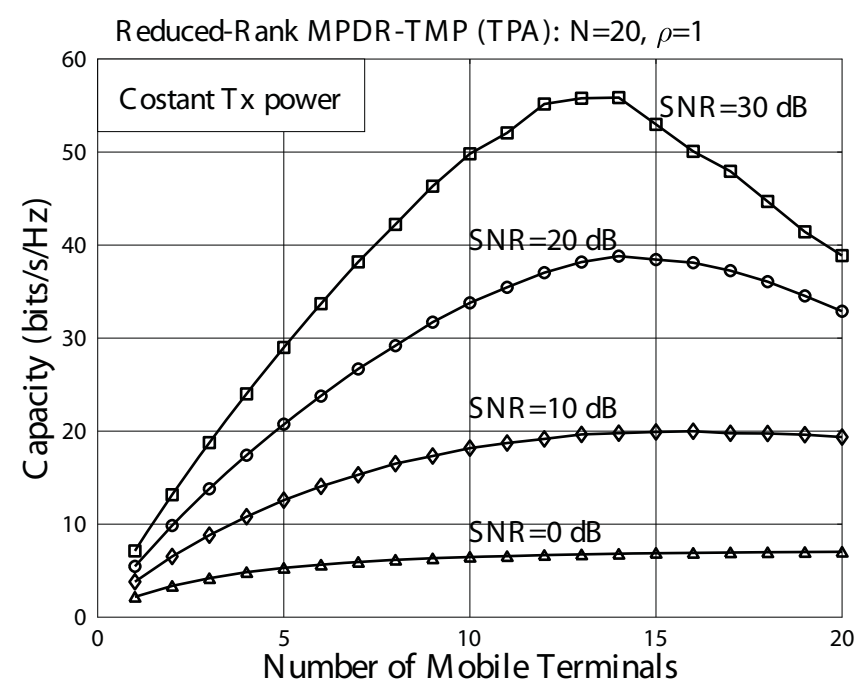

Fig. 6. Reduced-rank (TPA): Capacity versus number of MTs $(K)$ performance of the MPDR-TMP assisted SDMA using $N=20$ BS transmit antennas, when communicating over Rayleigh fading channels and assuming that the transmission power is constant regardless of the number of MTs supported.

sensitive to the varying of the noise-suppression factor in the preprocessing matrix (vectors). This observation in turn implies that the error and capacity performance of the SDMA system using MPDR-TMP is robust to the estimation error of the noise variance. Furthermore, it can be implied that, in a channel reciprocal SDMA system, when the noise powers of both the uplink and downlink is not significantly different, all the requirements, including the auto-correlation matrix, the subspace for reduced-rank preprocessing, etc., for downlink preprocessing may be directly extracted from the uplink.

Having shown the characteristic and performance of the SDMA systems using full-rank MPDR-TMP, below we provide the results for the SDMA systems using reduced-rank MPDR-TMP. Fig. 5 shows the achievable capacity versus the rank $L$ of the preprocessing subspace for the SDMA systems using the three types of reduced-rank MPDR-TMPs considered in Section IV. Specifically, the two best points seen in Fig. 3, i.e., the points corresponding to $(\mathrm{SNR}=20 \mathrm{~dB}$, $K=15)$ and $(\mathrm{SNR}=30 \mathrm{~dB}, K=17)$, were considered. From Fig. 5 we can see that both the PC- and CSM-based rank-reduction schemes are capable of reaching the full-rank capacity regardless of the SNR value, provided that the rank of the preprocessing subspace, ie. $\boldsymbol{P}_{k}$, reaches the number of MTs or the rank of the signal subspace. However, when the rank of the preprocessing subspace is lower than the number of MTs, there is a significant loss of capacity in comparison with the full-rank MPDR-TMP. By contrast, the achievable capacity of the TPA-based reduced-rank MPDR-TMP seems depends on the SNR value. As shown in Fig. 5, when SNR=20dB, the TPA-based scheme is capable of achieving a capacity that is very close to the full-rank MPDR-TMP. However, when $\mathrm{SNR}=30 \mathrm{~dB}$, the achieved capacity is lower than that achieved by the full-rank MPDR-TMP. When comparing the capacity achieved by the TPA-based scheme with that achieved by the PC- and CSM-based schemes, it can be shown that, for an available rank value, the TPA-based scheme is capable of reaching a significantly higher capacity than both the PCand CSM-based schemes. Furthermore, Fig. 5 shows that the CSM-based scheme outperforms the PC-based scheme in terms of the capacity of a given rank.

Fig.5 also implies that, for any given $K$ and SNR values, both the PC- and CSM-based schemes are capable of achieving the full-rank capacity. However, this is not always correct for the TPA-based MPDR-TMP. Fig. 6 shows the capacity of the TPA-based reduced-rank MPDR-TMP versus the number of MTs supported. In our simulations we assumed that the preprocessing subspace always used an optimum rank, which is usually very low. When comparing the results in Fig. 6 with that in Fig. 3 and Fig.5, we find that the TPA-based scheme reaches its highest capacity earlier than the full-rank scheme and also than the PC- and CSM-based schemes. Specifically, for $\mathrm{SNR}=30 \mathrm{~dB}$, the TPA-based scheme reaches its highest capacity when the number of MTs is $K=13$ or 14 . By contrast, the other schemes reach their highest capacity, when the number of MTs is $K=17$, as seen in Fig. 3 and Fig.5. Furthermore, Fig. 6 shows that the TPA-based scheme is capable of reaching the full-rank capacity, when the SNR is relatively low $(<20 \mathrm{~dB})$, while there is a gap from the full-rank capacity, when the SNR is relatively high, such as SNR=30dB.

Finally, in Figs. 7 - 10 the BER performance of the three types of reduced-rank MPDR-TMPs is investigated. The parameters used in our simulations are shown in the corresponding figures. From the results of Figs. 7 - 10, we may have the following observations.

- For both the PC- and CSM-based reduced-rank MPDRTMPs, the BER performance improves, when increasing the rank, $L$, of the preprocessing subspace, provided that the rank $L$ is lower than that of the signal subspace, which is the number of MTs supported. When the rank $L$ of the preprocessing subspace reaches the rank of the signal subspace, both reduced-rank MPDR-TMPs are capable of achieving the BER performance of the full-rank MPDRTMP.

- For both the PC- and CSM-based schemes, when the rank 
Reduced-rank MPDR-TMP (PC): N=20, K=10, $\rho=1$

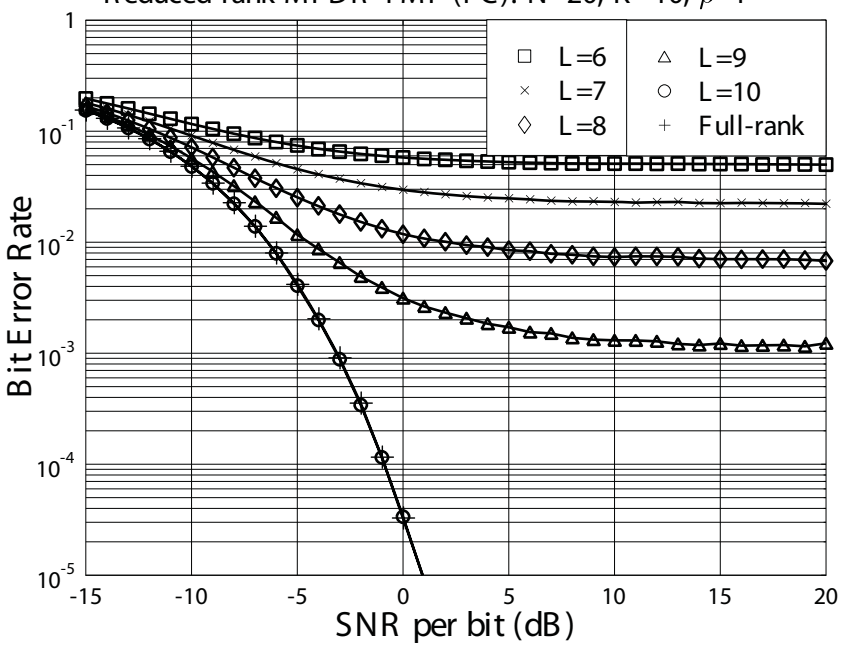

Fig. 7. Reduced-rank (PC): BER versus SNR per bit performance of the MPDR-TMP assisted SDMA using $N=20$ BS transmit antennas and supporting $K=10$ downlink MTs, when communicating over Rayleigh fading channels.

Reduced-rank MPDR-TMP (CSM): N=20, $K=10, \rho=1$

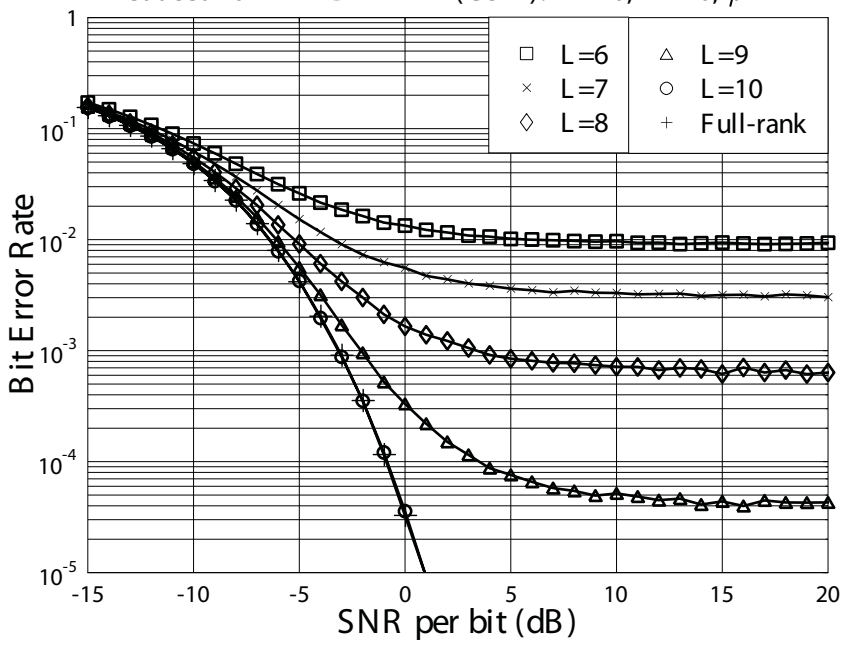

Fig. 8. Reduced-rank (CSM): BER versus SNR per bit performance of the MPDR-TMP assisted SDMA using $N=20 \mathrm{BS}$ transmit antennas and supporting $K=10$ downlink MTs, when communicating over Rayleigh fading channels.

$L$ of the preprocessing subspace is lower than that of the signal subspace, BER error floors are observed.

- It can be observed that, for any given rank $L(<K)$ of the preprocessing subspace, the CSM-based reducedrank MPDR-TMP outperforms the PC-based reducedrank MPDR-TMP.

- Finally, the TPA-based reduced-rank MPDR-TMP outperforms both the PC- and CSM-based reduced-rank MPDR-TMPs and achieves the best BER performance for a given rank $L$ of the preprocessing subspace. From the results of Figs.9 and 10, we can observe that the TPAbased reduced-rank MPDR-TMP is capable of achieving the BER performance very close to the full-rank BER performance with a rank $L$ that is significantly lower than the rank of the signal subspace. Specifically, as shown in Figs.9 and 10, when the target BER is in the range

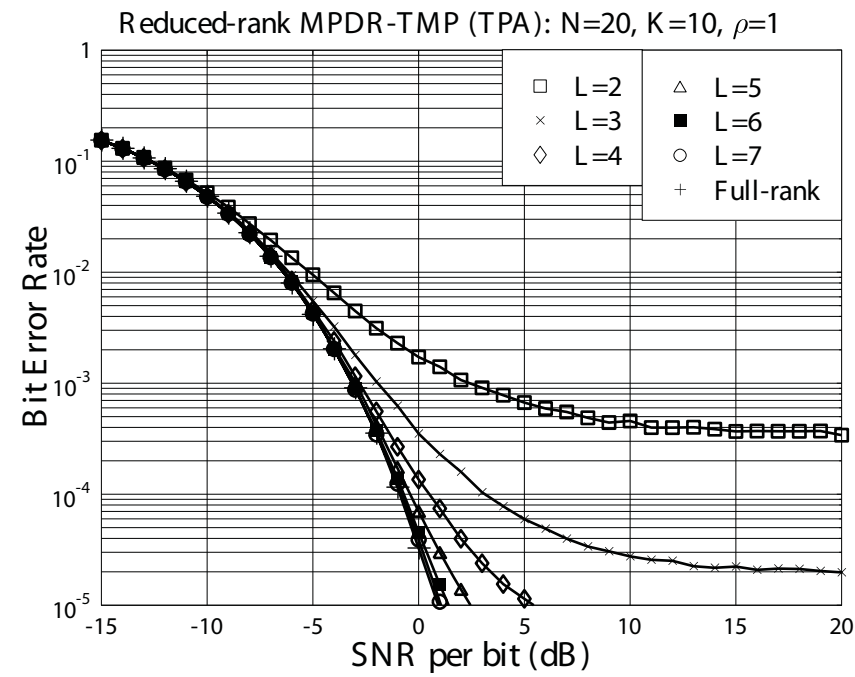

Fig. 9. Reduced-rank (TPA): BER versus SNR per bit performance of the MPDR-TMP assisted SDMA using $N=20 \mathrm{BS}$ transmit antennas and supporting $K=10$ downlink MTs, when communicating over Rayleigh fading channels.

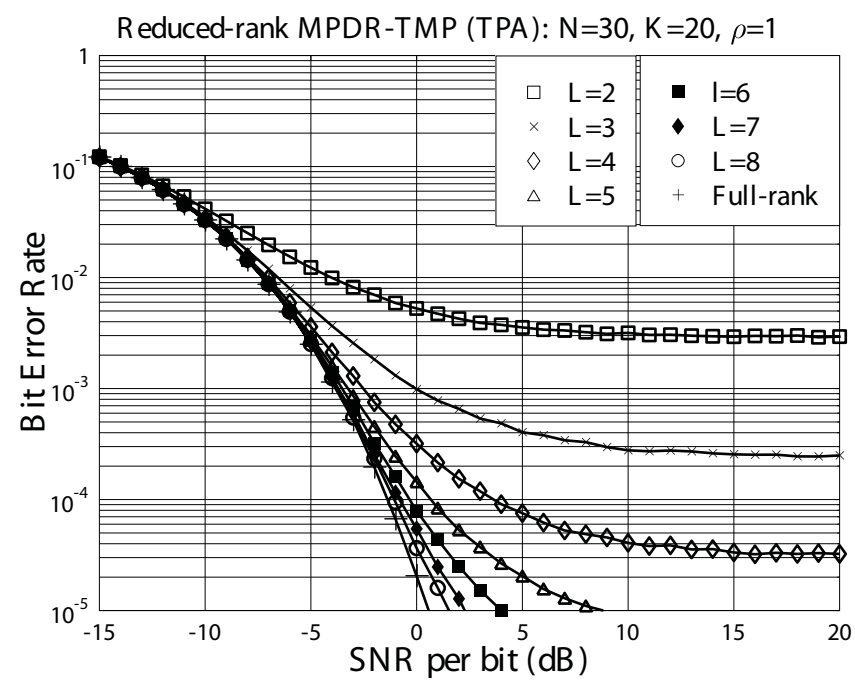

Fig. 10. Reduced-rank (TPA): BER versus SNR per bit performance of the MPDR-TMP assisted SDMA using $N=30 \mathrm{BS}$ transmit antennas and supporting $K=20$ downlink MTs, when communicating over Rayleigh fading channels.

of $\left(10^{-3}, 10^{-2}\right)$, a rank of $L=4$ or 5 is sufficient for achieving the full-rank BER performance, even the signal subspace has a rank of $K=10$ or 20 , respectively.

\section{CONCLUSIONS}

In this contribution a TMP scheme based on the MPDR principles has been proposed for the downlink multiuser MIMO systems. Its performance has been investigated in terms of the achievable capacity and BER. Our analysis and performance results show that in a MIMO system using the proposed MPDR-TMP the achievable capacity is capable of scaling linearly with the number of receive antennas (or the number of MTs supported), when the number of receive antennas is lower than the number of BS transmit antennas and provided that the total transmission power is sufficiently high. The MIMO systems using either the PC- or CSM-based 
reduced-rank MPDR-TMP are capable of reaching the fullrank capacity, provided that the preprocessing subspace has the same rank as the signal subspace. The MIMO systems using the TPA-based reduced-rank MPDR-TMP can reach a very high capacity with a rank which may be significantly lower than that of the signal subspace. However, when the total transmission power is high, the TPA-based technique may not reach the full-rank capacity. When considering the error performance, our simulation results show that the TPAbased scheme is the best among the rank-reduction techniques considered. It is capable of achieving the near full-rank BER performance with a preprocessing subspace rank, which is significantly lower than the rank of the signal subspace.

\section{ACKNOWLEDGEMENT}

The author would like to acknowledge with thanks the financial assistance from EPSRC of UK, and the anonymous reviewers for their helpful comments and valuable suggestions.

\section{REFERENCES}

[1] D. Gerlach and A. Paulraj, "Adaptive transmitting antenna arrays with feedback," IEEE Signal Processing Lett., vol. 1, pp. 150-152, Oct. 1994.

[2] R. Esmailzadeh, E. Sourour, and M. Nakagawa, "Prerake diversity combining in time-division duplex CDMA mobile communications," IEEE Trans. Veh. Technol., vol. 48, pp. 795-801, May 1999.

[3] B. Vojcic and W. Jang, "Transmitter preprocessing in synchronous multiuser communications," IEEE Trans. Commun., vol. 46, pp. 13461355, Oct. 1998.

[4] L.-U. Choi and R. D. Murch, "Transmit-preprocessing technique with simplified receivers for the downlink of MISO TDD-CDMA systems," IEEE Trans. Veh. Technol., vol. 53, pp. 285-295, Mar. 2004.

[5] R. L.-U. Choi and R. D. Murch, "New transmit schemes and simplified receivers for MIMO wireless communication systems," IEEE Trans. Wireless Commun., vol. 2, pp. 1217-1230, Nov. 2003.

[6] M. Joham, W. Utschick, and J. A. Nossek, "Linear transmit processing in MIMO communications systems," IEEE Trans. Signal Processing, vol. 53, pp. 2700-2712, Aug. 2005.

[7] L.-L. Yang, "A zero-forcing multiuser transmitter preprocessing scheme for downlink communications," IEEE Trans. Commun., vol. 56, no. 6, pp. 862-865, June 2008.

[8] R. Esmailzadeh and M. Nakagawa, "Pre-RAKE diversity combination in direct-sequence spread spectrum mobile communications systems," IEICE Trans. Commun., vol. E76-B, pp. 1008-1015, Aug. 1993.

[9] R. L.-U. Choi, K. B. Letaief, and R. D. Murch, "MISO CDMA transmission with simplified receiver for wireless communication handsets," IEEE Trans. Commun., vol. 49, pp. 888-898, May 2001.

[10] L.-U. Choi and R. D. Murch, "A transmit preprocessing technique for multiuser MIMO systems using a decomposition approach," IEEE Trans. Wireless Commun., vol. 3, pp. 20-24, Jan. 2004.

[11] L.-U. Choi and R. D. Murch, "A transmitter MIMO scheme with frequency domain pre-equalization for wireless frequency selective channels," IEEE Trans. Wireless Commun., vol. 3, pp. 929-938, May 2004.

[12] J. Choi and S. Perreau, "MMSE multiuser downlink multiple antenna transmission for CDMA systems," IEEE Trans. Signal Processing, vol. 52, pp. 1564-1573, June 2004.

[13] D. Reynolds, X. Wang, and K. N. Modi, "Interference suppression and diversity exploitation for multiantenna CDMA with ultra-low complexity receivers," IEEE Trans. Signal Processing, vol. 53, pp. 3226-3236, Aug. 2005.
[14] L.-L. Yang, "Design linear multiuser transmitters from linear multiuser receivers," in Proc. IEEE ICC'2007, June 2007.

[15] S. Verdu, Multiuser Detection. Cambridge University Press, 1998.

[16] H. L. V. Trees, Optimum Array Processing. Wiley Interscience, 2002.

[17] S. Haykin, Adaptive Filter Theory. Upper Saddle River, NJ: Prentice Hall, 3 ed., 1996.

[18] J. S. Goldstein and I. S. Reed, "Subspace selection for partially adaptive sensor array processing," IEEE Trans. Aerospace Electronic Syst., vol. 33, pp. 539-544, Apr. 1997.

[19] J. S. Goldstein and I. S. Reed, "Reduced-rank adaptive filtering," IEEE Trans. Signal Processing, vol. 45, pp. 492-496, Feb. 1997.

[20] J. R. Guerci, J. S. Goldstein, and I. S. Reed, "Optimal and adaptive reduced-rank STAP," IEEE Trans. Aerospace Electronic Syst., vol. 36, pp. 647-663, Apr. 2000.

[21] J. S. Goldstein, I. S. Reed, and L. L. Scharf, "A multistage representation of the Wiener filter based on orthogonal projections," IEEE Trans. Inform. Theory, vol. 44, pp. 1943-2959, Nov. 1998.

[22] M. L. Honig and W. Xiao, "Performance of reduced-rank linear interference suppression," IEEE Trans. Inform. Theory, vol. 47, pp. 1928-1946, July 2001.

[23] M. L. Honig and J. S. Goldstein, "Adaptive reduced-rank interference suppression based on the multistage Wiener filter," IEEE Trans. Commun., vol. 50, pp. 986-994, June 2002.

[24] S. Moshavi, E. G. Kanterakis, and D. L. Schilling, "Multistage linear receivers for DS-CDMA systems," International J. Wireless Inform. Networks, vol. 3, pp. 1-17, Jan. 1996.

[25] X. Wang and H. V. Poor, Wireless Communication Systems-Advanced Techniques for Signal Reception. Prentice Hall, 2003.

[26] T. M. Cover and J. A. Thomas, Elements of Information Theory. New York: John Wiley \& Sons, 1991.

[27] L.-L. Yang, "Reduced-rank MMSE detection in space-time coded spacedivision multiple-access systems," in Proc. IEEE PIMRC'06, Sept. 2006.

[28] R. R. Muller and S. Verdu, "Design and analysis of low-complexity interference mitigation on vector channels," IEEE J. Select. Areas Commun., vol. 19, pp. 1429-1441, Aug. 2001.

[29] J. Brehmer and et al., "Reduced-complexity linear and nonlinear precoding for frequency-selective MIMO channels," in Proc. IEEE VTC'2004 Fall, pp. 3684-3688, Sept. 2004.

[30] W. Chen, U. Mitra, and P. Schniter, "On the equivalence of three reduced rank linear estimators with applications to DS-CDMA," IEEE Trans. Inform. Theory, vol. 48, pp. 2609-2614, Sept. 2002.

[31] I. E. Telatar, "Capacity of multiantenna Gaussian channels," European Trans. Telecommun., vol. 10, pp. 585-595, Nov./Dec. 1999.

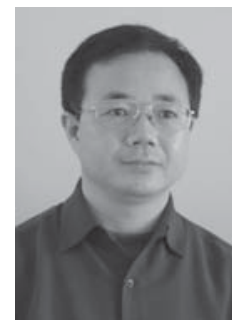

Dr. Lie-Liang Yang received his B.Eng. degree in communication engineering from Shanghai TieDao University, Shanghai, China in 1988, and his M.Eng, $\mathrm{Ph} . \mathrm{D}$. degrees in communications and electronics from Beijing (Northern) Jiaotong University, Beijing, China in 1991 and 1997, respectively. From June 1997 to December 1997 he was a visiting scientist of the Institute of Radio Engineering and Electronics, Academy of Sciences of the Czech Republic. Since December 1997, he has been with the Communications Research Group, School of Electronics and Computer Science, University of Southampton, U.K, where he was first a Postdoctoral Research Fellow (Dec. 1997 - Aug. 2002), then a Lecturer (Sept. 2002 - Feb. 2006), and currently holds the academic post of Readership. Dr. Yang's research has covered a wide range of areas in wireless communication, networking and signal processing. He has published over 200 research papers in journals and conference proceedings, authored/coauthored two books and also published several book chapters. He was awarded the Royal Society Sino-British Fellowship in 1997 and the EPSRC Research Fellowship in 1998. Dr. Yang is currently an associate editor for the JOURNAL of COMmunications and Networks (JCN), the Journal of COMmunications (JCM) and SeCURITy AND COMMUniCATION Networks JOURNAL (SCN). 